\title{
Comparison of Partial Citrate Synthase Gene (gltA) Sequences for Phylogenetic Analysis of Bartonella Species
}

\author{
RICHARD J. BIRTLES* AND DIDIER RAOULT \\ Unité des Rickettsies, Centre National de la Recherche Scientifique EP J0054, Faculté de Médecine, \\ 13385 Marseille Cédex 5, France
}

\begin{abstract}
Nucleotide base sequence data were obtained for a 940-bp fragment of the citrate synthase-encoding gene (gltA) of representatives of the eight validly described Bartonella species and seven uncharacterized Bartonella strains obtained from small mammals. Complete 16S rRNA gene sequences were also determined for the uncharacterized strains, and these sequences revealed that each strain had a unique sequence which was very similar to the sequences of the previously recognized Bartonella species. A comparison of the gltA sequences of the different Bartonella species revealed that the levels of similarity between sequences were 83.8 to $93.5 \%$, whereas comparisons of sequences obtained from different strains of the same species revealed that the levels of similarity were more than $\mathbf{9 9 . 8 \%}$. One of the uncharacterized strains had a gltA sequence that matched the sequence of Bartonella elizabethae, three uncharacterized strains had sequences which were more than $99.6 \%$ similar to each other (but less than 93.5\% similar to any other sequence), and the remaining three uncharacterized strains each exhibited less than $\mathbf{9 3 . 5 \%}$ sequence similarity to other Bartonella species or isolates. Phylogenetic trees were inferred from multiple alignments of both gltA and 16S ribosomal DNA (rDNA) sequences. Whereas the proposed intra-Bartonella architecture of trees inferred from 16S rDNA sequence data by using both distance matrix and parsimony methods had virtually no statistical support, the trees inferred from the glt $A$ sequence data contained four well-supported lineages in the genus. The gltA-derived phylogeny appears to be more useful than the phylogeny derived from 16S rDNA sequence data for investigating the evolutionary relationships of Bartonella species, and the validity of the lineages identified by the gltA analysis is discussed in this paper.
\end{abstract}

The family Bartonellaceae has recently been subjected to considerable taxonomic reassessment, which has resulted in (i) the transfer of members of the genus Rochalimaea (formerly placed in the Rickettsiaceae) to the genus Bartonella (7), (ii) the unification of the two established genera in the family, the genera Bartonella and Grahamella, with the name of the former taking precedence (5), (iii) the proposal of several new species and subspecies $(5,6)$, and (iv) the removal of the family from the order Rickettsiales (7). The restructured family now includes the single genus Bartonella, which contains eight species. A polyphasic approach was used to develop all of these proposals, and phylogenetic data derived from a comparison of 16S rRNA gene sequences provided important supporting evidence. These studies also revealed that the Bartonella species are very closely related and that they exhibit very high levels of 16S rRNA gene sequence similarity $(>97.8 \%)$ and significant levels of DNA-DNA homology $(16$ to $67 \%)(5,7,8)$. The paucity of 16S rRNA gene sequence differences between species limits the usefulness of this tool for inferring intragenus phylogeny and, as in other closely related taxa, does not allow measurement of small phylogenetic distances with sufficient precision to define species $(14,29)$.

Although 16S rRNA gene sequence analysis remains the most widely used tool in bacterial phylogeny and in most instances the data concur with the results of phenotypic and genotypic analyses, workers have expressed concern that if a single gene is used as the sole basis of phylogeny, it is possible that a recent transfer of the gene between dissimilar species could give rise to an organism that appears to be specifically

* Corresponding author. Mailing address: Unité des Rickettsies, CNRS EP J0054, Faculté de Médecine, 27, Boulevard Jean Moulin, 13385 Marseille Cédex 5, France. Phone: (33) 918343 75. Fax: (33) 91 830390 . Electronic mail address: raoult@pacwan.mm-soft.fr. related to the donor of the gene rather than to its true relatives (27). One way of revealing such events is to compare phylogenetic trees inferred from genetic sequences independent of one another, genes which have not been recently acquired will reveal the same phylogeny (17). In a recent study, phylogenetic trees inferred from a comparison of partial methyl-coenzyme $\mathrm{M}$ reductase subunit $\mathrm{A}$ gene $(\mathrm{mcrl})$ sequences from closely related species belonging to the family Methanosarcinaceae corresponded closely to trees inferred from $16 \mathrm{~S}$ rRNA gene sequence data (28). In this study it was also determined that mcrI sequences evolved about three times more rapidly than 16S rRNA gene sequences; thus, closely related species exhibited markedly lower levels of sequence similarity. An increase in the number of discenible differences between such species should allow more confident estimates of their phylogenetic relationships.

Citrate synthase is the first enzyme of the tricarboxylic acid cycle and thus is a key regulator of intracellular ATP production in both prokaryotic and eucaryotic cells (32). Although this enzyme exists in two multimeric forms (a dimer and a sexmer), monomers of the two forms are similar in size and exhibit sequence homology (32). The nucleic acid sequence of the glt $A$ gene, which codes for the enzyme, has been determined for a number of bacteria, including Bartonella henselae $(9,19-21,33)$. The use of glt $A$ comparisons to estimate divergence among closely related species was first proposed by Regnery and his colleagues, who demonstrated genotypic relationships among Rickettsia species by comparing multiple restriction endonuclease digestion patterns of partial gltA PCR amplicons (24), and identification schemes developed from this work were subsequently used to differentiate Bartonella species (23). Recently, 345-bp nucleotide sequences of these gltA amplicons have been determined for five Bartonella species (16), and a comparison of these sequences revealed levels of similarity between approximately 80 and $90 \%$, 
TABLE 1. Sources of Bartonella isolates

\begin{tabular}{|c|c|c|c|}
\hline Species & Strain & $\begin{array}{l}\text { EMBL and GenBank 16S } \\
\text { rRNA sequence accession no. }\end{array}$ & Source (reference) ${ }^{a}$ \\
\hline B. bacilliformis & LA6.3 & $\mathrm{Z} 70003$ & Blood of bartonellosis patient, Yumpe, Huaillacayán Valley, Ancash, Peru \\
\hline B. doshiae & $\mathrm{R} 18^{\mathrm{T}}$ & Z31351 & NCTC 12862 \\
\hline B. elizabethae & $\mathrm{F} 9251^{\mathrm{T}}$ & L01260 & ATCC 49927 \\
\hline B. grahamii & $\mathrm{V} 2^{\mathrm{T}}$ & Z31349 & NCTC 12860 \\
\hline B. henselae & Houston- $1^{\mathrm{T}}$ & M73229 & ATCC 49882 \\
\hline B. henselae & WC1 & & Blood of domestic cat, London, United Kingdom (10) \\
\hline B. henselae & URLLY 8 & & Cat scratch disease patient $(10)$ \\
\hline B. henselae & URLIE 9 & & Endocarditis patient $(10)$ \\
\hline B. quintana & Fuller $^{\mathrm{T}}$ & M73228 & ATCC VR-358 \\
\hline B. quintana & Oklahoma & & Gift from D. F. Welch, University of Oklahoma, Oklahoma City \\
\hline B. quintana & Paris-1 & & Endocarditis patient (11) \\
\hline B. quintana & Marseille-1 & & Chronic adenopathy patient (22) \\
\hline B. taylorii & $\mathrm{M}^{\mathrm{T}}$ & Z31350 & NCTC 12861 \\
\hline B. vinsonii & Baker $^{T}$ & Z31352 & ATCC VR-152 \\
\hline Bartonella sp. & C1-phy & Z70006 & Blood of Phyllotis sp., Huaillacayán Valley, Ancash, Peru \\
\hline Bartonella sp. & C4-phy & Z70007 & Blood of Phyllotis sp., Huaillacayán Valley, Ancash, Peru \\
\hline Bartonella sp. & C5-rat & Z70008 & Blood of Rattus sp., Huaillacayán Valley, Ancash, Peru \\
\hline Bartonella sp. & C7-rat & Z70004 & Blood of Rattus sp., Huaillacayán Valley, Ancash, Peru \\
\hline Bartonella sp. & R-phy1 & Z70005 & Blood of Phyllotis sp., Rimac Valley, Lima, Peru \\
\hline Bartonella sp. & R-phy2 & Z70001 & Blood of Phyllotis sp., Rimac Valley, Lima, Peru \\
\hline Bartonella sp. & $\mathrm{N} 40$ & Z70002 & Blood of Apodemus sylvaticus, Staffordshire, United Kingdom \\
\hline
\end{tabular}

${ }^{a}$ NCTC, National Collection of Type Cultures, Central Public Health Laboratory, London, United Kingdom; ATCC, American Type Culture Collection, Rockville, Md.

values that were markedly lower than the levels of similarity between 16S rRNA genes. In this study, 946-bp sequences, representing more than $75 \%$ of the complete gene, were determined for representatives of all eight Bartonella species and for an additional seven Bartonella strains which could be distinguished from these species on the basis of 16S rRNA sequence data. The selection of sequencing primers was simplified by referring to the glt $A$ sequence of $B$. henselae (20). The glt $A$ sequences obtained were analyzed and were used to create phylogenetic trees, which were compared with 16S rRNA trees.

\section{MATERIALS AND METHODS}

Bacterial strains and culture conditions. The sources of the Bartonella strains used in this study are shown in Table 1. The strains isolated from the blood of small mammals in Peru were obtained as part of a concurrent study through collaboration with Humberto Guerra, Alejandro Llanos-Cuentas, and Kike Perez and their staff at the Instituto de Medicina Tropical Alexander Von Humboldt, University Cayatana Herrer, Lima, Peru. Strain N40 was obtained during a previous study (1). The previously uncharacterized strains were preliminarily identified as bartonellae on the basis of their phenotypic properties, including their growth requirements (the strains could not be cultivated on media lacking blood), colonial morphology, microscopic appearance (weakly staining gram-negative rods), and lack of catalase and oxidase activities, and the results of a restriction endonuclease analysis of their amplified 16S rRNA genes (2).

All strains were grown on Columbia blood agar containing $5 \%$ whole sheep blood. Most plates were incubated at $37^{\circ} \mathrm{C}$ in a $\mathrm{CO}_{2}$-rich, moist atmosphere for 10 days; the exceptions were the plates inoculated with Bartonella bacilliformis, which were incubated at $30^{\circ} \mathrm{C}$. Crude DNA extracts that were suitable for use as templates in PCR were prepared from isolates by using a Chelex 100 reagent (30).

16S rRNA gene amplification, purification, and sequence determination. The 16S rRNA genes were amplified by PCR under conditions which have been described previously (3). PCR amplicons were purified by using a QIAquick PCR purification kit (Qiagen $\mathrm{GmbH}$, Hilden, Germany) according to the manufacturer's instructions for use as templates in cycle sequencing reactions. For sequencing reactions we used the reagents of an Amplicycle kit (Pharmacia Biotech, St-Quentin Yveline, France), which included fiuorescein-labelled primers (Eurogentec, Seraing, Belgium), according to the manufacturer's instructions. The primers used were universal eubacterial 16S rRNA sequencing primers that have been described previously (12). The sequencing products were resolved on 0.35 -mm-thick READIMIX acrylamide gels (Pharmacia), and fluorescein-labelled products were detected and translated into sequence data by using a model ALF automated sequencer (Pharmacia) and associated software.
Amplification of gltA. PCR amplification of a 1,038-bp fragment of gltA was carried out by using an MJ Research model PTC-200 thermal cycler. Each reaction mixture contained $2 \mu$ l of crude DNA extract, 40 pmol of each primer, $1 \mu \mathrm{l}$ of a deoxynucleoside triphosphate mixture (dATP, dCTP, dGTP, and dTTP, each at a concentration of $10 \mathrm{mM}$ ), $10 \mu \mathrm{l}$ of $10 \times$ reaction buffer, $8 \mu$ l of a $25 \mathrm{mM}$ $\mathrm{MgCl}_{2}$ solution, and $1 \mathrm{U}$ of AmpliTaq DNA polymerase (Perkin-Elmer Cetus, Norwalk, Conn.); the volume of the reaction mixture was adjusted to $100 \mu \mathrm{l}$ with sterile distilled water. The primers used were primer CSi40f $\left(5^{\prime}\right.$-TTACTTATG ATCCKGGYTTTA, where $\mathrm{K}$ is equimolar amounts of $\mathrm{G}$ and $\mathrm{T}$ and $\mathrm{Y}$ is equimolar amounts of $\mathrm{C}$ and $\mathrm{T}$ ) and primer BhCS.1137n (20). The thermal cycle for amplification consisted of 35 cycles, with each cycle consisting of denaturation at $95^{\circ} \mathrm{C}$ for $10 \mathrm{~s}$, primer annealling at $50^{\circ} \mathrm{C}$ for $20 \mathrm{~s}$, and primer extension at $72^{\circ} \mathrm{C}$ for $50 \mathrm{~s}$. Amplification products were resolved by $1 \%$ agarose gel electrophoresis and were visualized under UV light following ethidium bromide staining.

Purification and sequencing of gltA amplicons. The glt $A$ amplicons were purified as described above by using QIAquick columns, and the purified products were sequenced by using the reagents of an Amplicycle kit (Pharmacia Biotech, Uppsala, Sweden) and fluorescein-labelled primers (Eurogentec) according to the manufacturer's instructions. The following primers were used for sequencing: primers CS140f, CS443f (5'-GCTATGTCTGCATTCTATCA), CSH1f (5'-GCG AATGAAGCGTGCCTAAA), and BhCS.1137n. Sequencing products were resolved and translated into sequence data as described above.

Analysis of sequence data and construction of phylogenetic trees. For each strain tested, the complete primary sequences of 16S rRNA genes and glt $A$ were assembled by alignment and by combining the sequences generated by each primer, using DNASIS (Hitachi Software Engineering America, Ltd., Brisbane, Calif.). The $16 \mathrm{~S}$ rRNA sequence data obtained were confirmed by examining base pairing in the predicted secondary structure, and glt $A$ sequences were assessed by identifying codon triplets.

Complete primary $16 \mathrm{~S}$ rRNA sequences were then aligned with each other and with 16S rRNA sequences of the recognized Bartonella species and other members of the Eubacteria by using version V of the CLUSTAL multisequence alignment program (15). The glt $A$ sequences obtained in this study were aligned with each other, with the sequence of $B$. henselae, and with the sequences available for other members of the Eubacteria by using the same method. A total of 100 bootstrap samples for both alignments were produced by using the program SEOBOOT from the PHYLIP version 3.4 suite of software (13), and a phylogenetic tree was inferred from each bootstrap sample by using parsimony (DNAPARS in PHYLIP). The resulting trees were combined to yield a consensus tree (CONSENSE in PHYLIP). A matrix of evolutionary distances was also derived from each bootstrap alignment by using DNADIST (PHYLIP) and the assumptions of Jukes and Cantor. Trees were inferred from the matrices by using the FITCH program (PHYLIP), in which a least-squares method is used to construct a phylogenetic tree that best fits the data. The resulting trees were combined to yield a consensus tree (CONSENSE in PHYLIP).

Levels of similarity between $g l t A$ sequences were determined by sequentially comparing pairs by using the DNA homology search function of DNASIS. 
TABLE 2. Levels of $16 \mathrm{~S}$ rRNA gene sequence similarity and glt $A$ sequence similarity

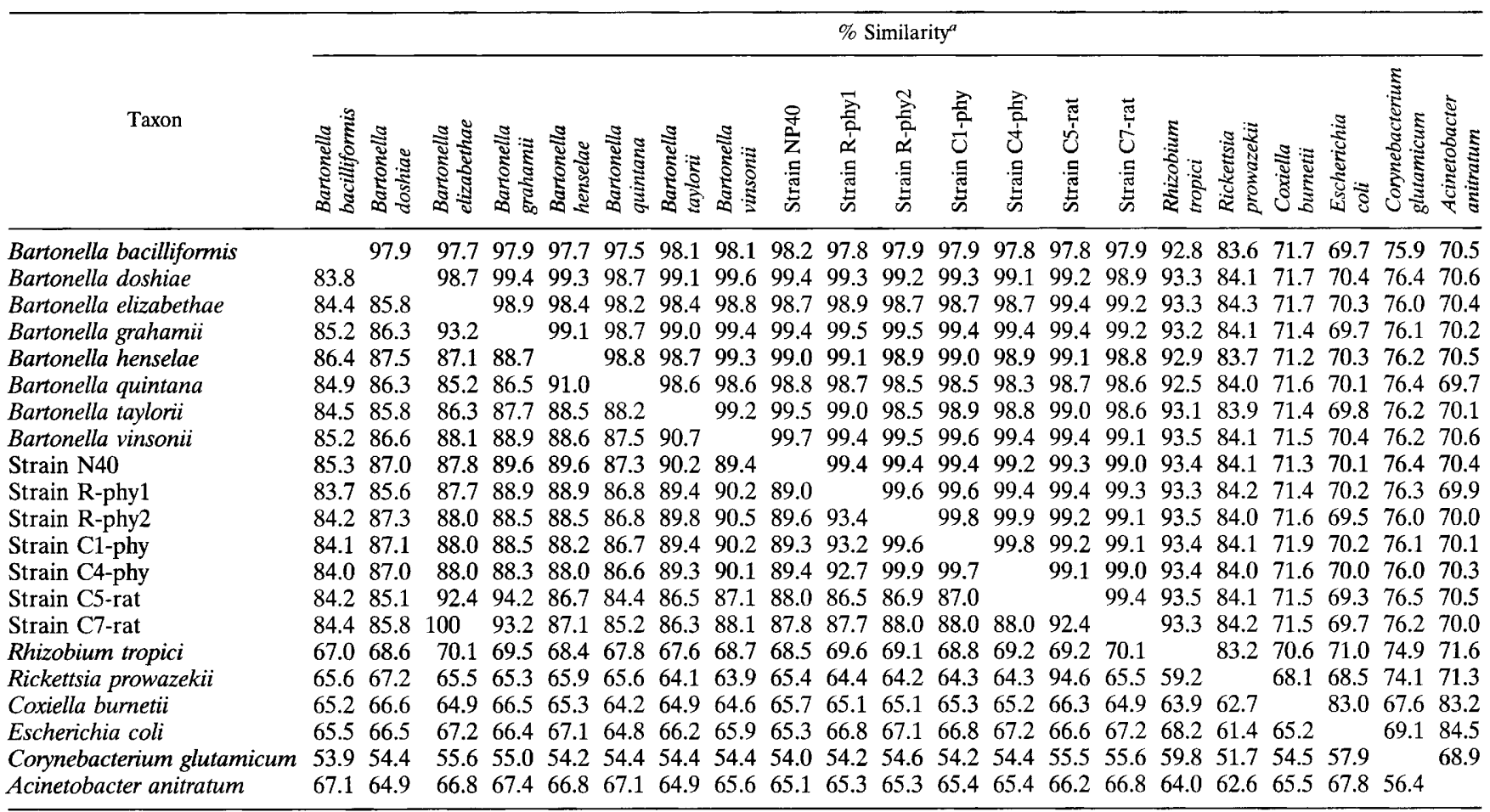

\footnotetext{
${ }^{a}$ The values on the upper right are levels of $16 \mathrm{~S}$ rRNA gene sequence similarity, and the values on the lower left are levels of gltA gene sequence similarlity.
}

Nucleotide sequence accession numbers. The 16S rRNA gene sequences which we determined have been deposited in the EMBL data bank under the following accession numbers: strain C1-phy, Z70006; strain C4-phy, Z70007; strain C5-rat, Z70008; strain C7-rat, Z70004; strain R-phy1, Z70005; strain Rphy2, Z70001; strain N40, Z70002; and B. bacilliformis LA6.3, Z70003. The $16 \mathrm{~S}$ rRNA gene sequence accession numbers for the eight currently recognized Bartonella species are shown in Table 1, and the 16S rRNA gene sequence accession numbers for the organisms used for comparison are as follows: Rickettsia prowakezii, M21789; Acinetobacter anitratum, U10874; Corynebacterium glutamicum, X80629; Rhizobium tropici, U38469; Escherichia coli, Z46753; and Coxiella burnetii, M21291.

The gltA sequences which we determined have been deposited in the EMBL data bank under the following accession numbers: $B$. bacilliformis LA6.3, Z70021; Bartonella doshiae, Z70017; Bartonella elizabethae, Z70009; Bartonella grahamii, Z70016; Bartonella quintana Fuller ${ }^{\mathrm{T}}$ (T = type strain), Z70014; Bartonella taylorii, Z70013; Bartonella vinsonii, Z70015; strain C1-phy, Z70022; strain C4-phy, Z70019; strain C5-rat, Z70018; strain C7-rat, Z70020; strain R-phy1, Z70010; strain R-phy2, Z70011; and strain N40, Z70012. The gltA sequence accession numbers for the reference organisms are as follows: Rhizobium prowazekii, M17149; A. anitratum, M33037; Corynebacterium glutamicum, X71489; Rhizobium tropici, Z34516; E. coli, J01619; and Coxiella burnetii, L33409.

\section{RESULTS AND DISCUSSION}

Sequence alignment. The primary $16 \mathrm{~S}$ rRNA gene sequences generated for the seven uncharacterized Bartonella isolates studied consisted of between 1,409 and 1,411 bp; i.e., they were equivalent to almost the entire gene. The primary sequence generated for $B$. bacilliformis LA6.3 consisted of 1,380 bp. For the multiple alignment of 16S rRNA gene sequences we used the sequences of the seven uncharacterized strains (strains C1phy, C4-phy, C5-rat, C7-rat, R-phy1, R-phy2, and N40), B. bacilliformis LA6.3, the eight currently recognized Bartonella species, Rickettsia prowazekii, A. anitratum, Corynebacterium glutamicum, Rhizobium tropici, E. coli, and Coxiella burnetii (the last six species were chosen because glt $A$ data were available for them).

The primary glt $A$ sequences generated for the Bartonella strains were 946 bp long and represented the sequence be- tween positions 136 and 1082 of the $B$. henselae glt $A$ sequence, as determined previously (19). For the multiple alignment of glt $A$ sequences we used the sequences of $B$. bacilliformis LA6.3, B. doshiae, B. elizabethae, B. grahamii, B. quintana Fuller ${ }^{\mathrm{T}}, B$. taylorii, $B$. vinsonii, strains C1-phy, C4-phy, C5-rat, C7-rat, Rphy1, R-phy2, and N40, Rickettsia prowazekii, A. anitratum, Corynebacterium glutamicum, Rhizobium tropici, E. coli, and Coxiella burnetii.

Analysis of uncharacterized Bartonella isolates by comparison of 16S rRNA gene sequences. Phylogenetic inferences were made on the basis of a 1,327-bp multiple alignment of $16 \mathrm{~S}$ rRNA gene sequences from which regions of ambiguity (although not regions of hypervariability) had been removed. All seven uncharacterized Bartonella isolates had unique $16 \mathrm{~S}$ rRNA gene sequences, although all of these organisms exhibited very high levels of sequence similarity with each other and with the type strains of the eight Bartonella species (Table 2). There appears to be one region of the 16S rRNA gene which is highly variable in Bartonella species; this region corresponds to positions 183 to 187 ( $E$. coli structural model), in variable region V2. At this point, the sequences of the seven uncharacterized isolates have between one and three base insertions. An examination of $16 \mathrm{~S}$ rRNA secondary-structure models suggests that these changes should result in a slight lengthening of helix 10 or a slight enlargement of the loop that it carries. The hypervariability of this region has been observed previously in other Bartonella isolates, notably a strain of $B$. vinsonii which has a 27-bp insertion at this point (6).

An analysis of the phylogenetic tree (Fig. 1) derived from the distance matrix indicated that the seven uncharacterized strains belong to a monophyletic cluster which includes the eight recognized Bartonella species and no other organisms. Of the non-Bartonella species included in this study, the two representatives of the alpha subdivision of the Proteobacteria, to 


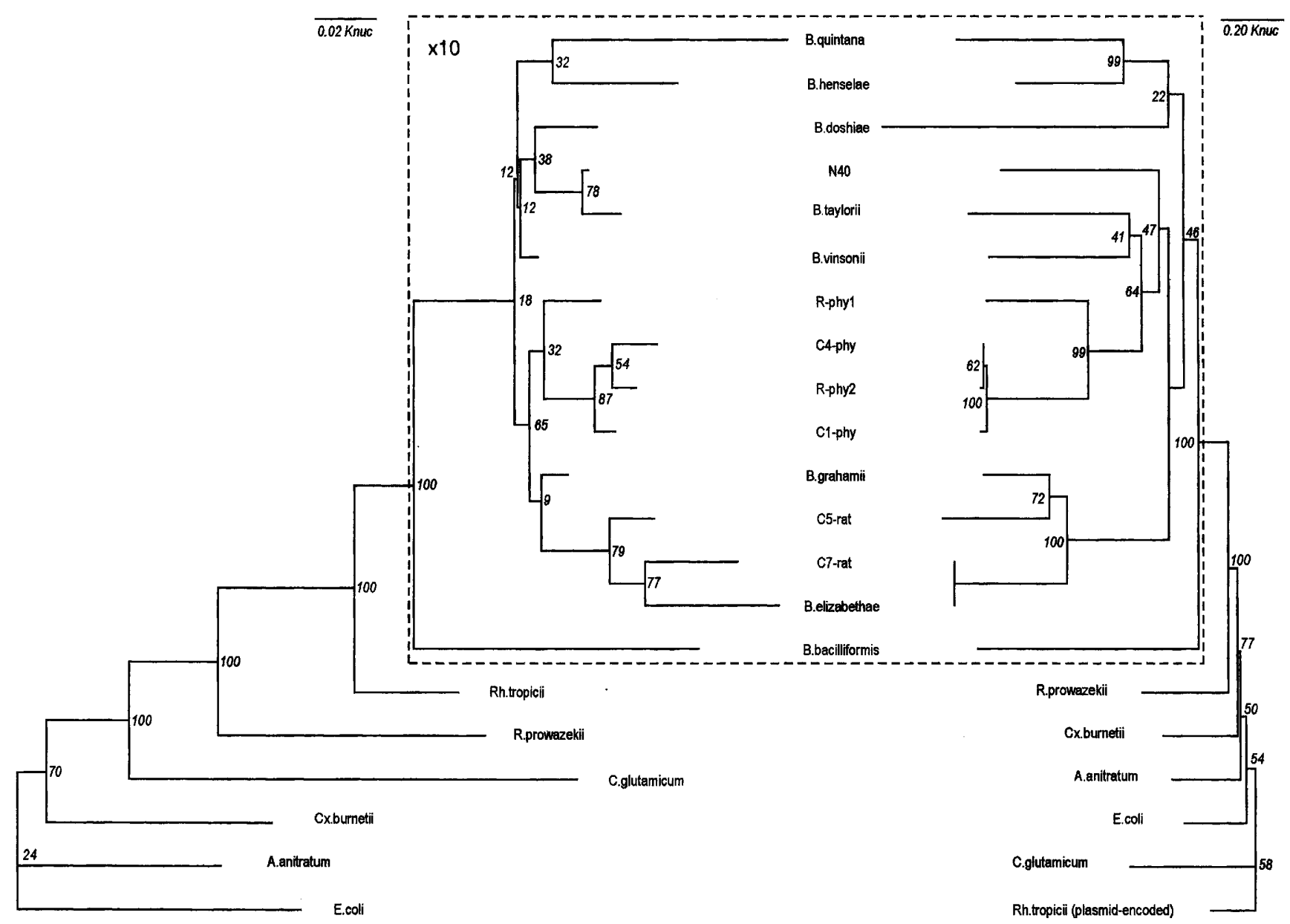

FIG. 1. Comparison of Fitch distance matrix trees derived from a 940-bp fragment of gltA (right side) and from the 16S rRNA gene (left side) for Bartonella strains and other representatives of the Eubacteria. The support for each branch, as determined from 100 bootstrap samples, is indicated by the value at the node. The lengths of vertical lines are not significant. The scale inside the dashed box is 10 times larger than the scale outside the box. Abbreviations: B., Bartonella; Rh., Rhizobium; R., Rickettsia; C., Corynebacterium; Cx., Coxiella; A., Acinetobacter; E., Escherichia.

which Bartonella species are also assigned, lie in closest evolutionary proximity. Within the Bartonella cluster, $B$. bacilliformis remains the most outlying species. Previously, it could have been proposed that the geographic isolation of this species (which is limited to the inter-Andean valleys of South America) influenced its divergence from the other Bartonella species; however, the six uncharacterized Bartonella strains which originated from the same region do not exhibit a closer evolutionary relationship to $B$. bacilliformis than to the other Bartonella species, which originated in Europe and the United States. The four isolates obtained from Phyllotis mice grouped together on the tree, although support for this cluster was only moderate ( 65 of 100 bootstrap samples). Although the isolates obtained from Peruvian rats also occur close to one another, they also exhibit high levels of homology with $B$. elizabethae, a species presently represented by a single isolate obtained from a patient with endocarditis in the United States (8). In general, the branching order of the tree has only moderate support, and the instability of the proposed structure was confirmed by the results of a parsimony analysis of the sequence data (Fig. 2). Other than the branch containing $B$. bacilliformis, no branch of the genus, on either tree, had the support of more than $90 \%$ of the bootstrap samples. Indeed, a comparison of the branching order of the Bartonella species on the two trees revealed marked differences. Conversely, outside the Bartonella cluster, the stability of the proposed branching order was considerably higher; when a distance matrix analysis was performed, four of the six branches were retained in more than $90 \%$ of bootstrap samples, whereas when a parsimony analysis was performed, all six branches had this level of support.

Although the finding described above may suggest that there are no distinct evolutionary subgroups in the genus Bartonella, it more likely emphasizes the insensitivity of $16 \mathrm{~S}$ ribosomal DNA (rDNA) sequence comparisons for inferring evolutionary relationships at this level. It is clear that inferences are difficult to make with confidence when there are so few base differences between sequences and when the base differences occur at highly variable positions where multiple base changes may have taken place.

Phylogeny of Bartonella species based on partial gltA sequences. A pairwise comparison of glt $A$ sequences revealed similarity values between 83.7 and $100 \%$ for the Bartonella strains (Table 2), whereas levels of similarity of less than $70 \%$ were observed between the sequences of Bartonella species and the sequences of organisms belonging to other genera. The sequences of the four isolates of $B$. henselae were virtually identical, differing at only one base site (Table 3 ); this level of similarity was also observed for the four strains of $B$. quintana 

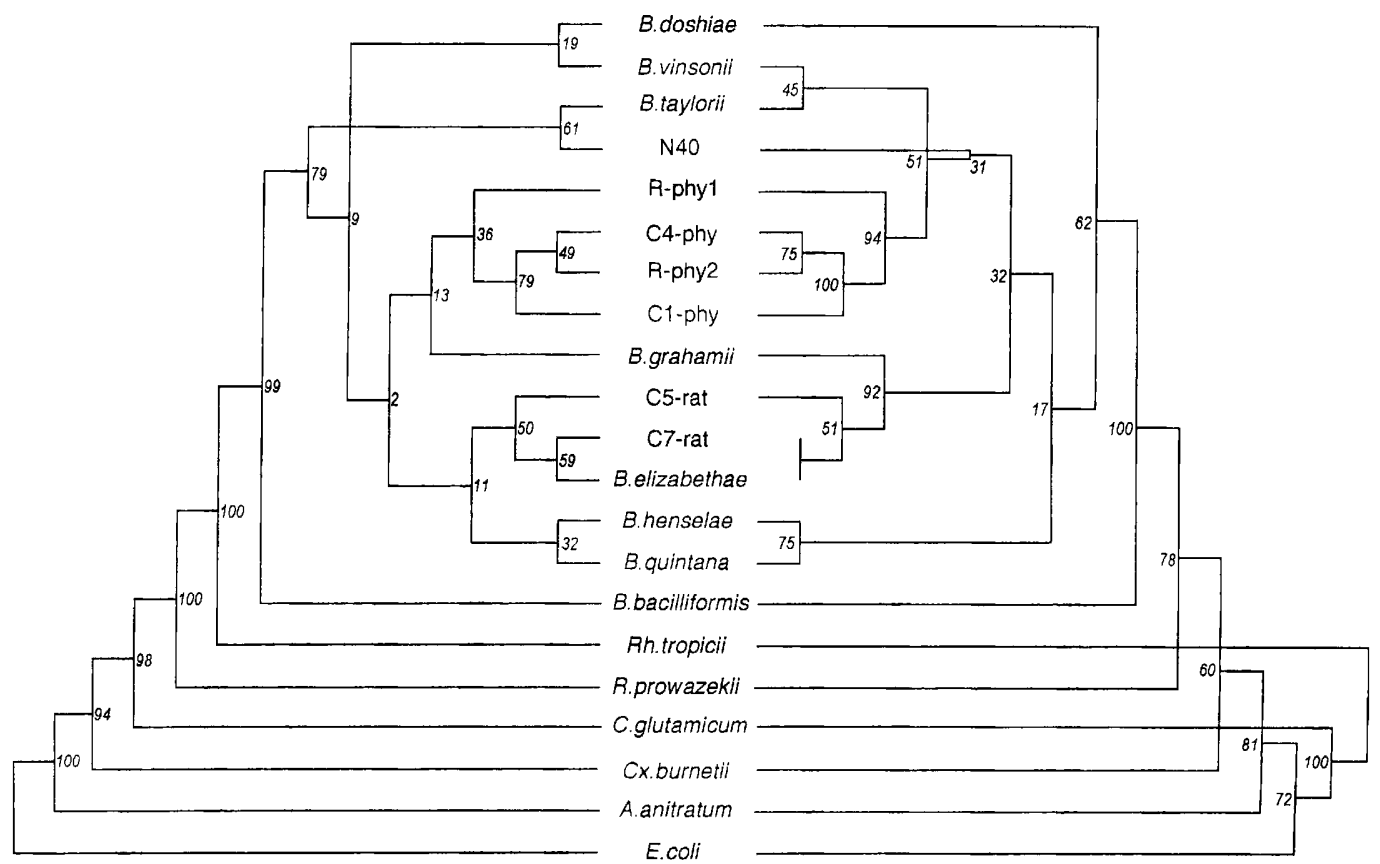

FIG. 2. Comparison of parsimony trees derived from a 940-bp fragment of gltA (right side) and from the 16S rRNA gene (left side) for Bartonella strains and other representatives of the Eubacteria. The support for each branch, as determined from 100 bootstrap samples, is indicated by the value at the node. This analysis provided tree topology only, and the lengths of both the vertical lines and the horizontal lines are not significant. Abbreviations: B., Bartonella; Rh., Rhizobium; R., Rickettsia; C., Corynebacterium; Cx., Coxiella; A., Acinetobacter, E., Escherichia.

tested (Table 3). The highest level of similarity between sequences from different Bartonella species was $93.2 \%$. These observations are consistent with the results of other genotypic analyses of Bartonella species; a comparison of the hypervariable spacer region sequence between the $16 \mathrm{~S}$ and $23 \mathrm{~S}$ rRNA genes revealed very high levels of similarity between strains of the same species (seven $B$. quintana strains differed at a maximum of 5 sites in a 1,331-bp sequence), whereas the differences between species were great enough that reasonable alignments could not be obtained (levels of similarity, less than $50 \%$ ) (25). A macrorestriction analysis of Bartonella strains in which pulsed-field gel electrophoresis was used also revealed that strains of the same species had very similar profiles which could be clearly differentiated from the profiles obtained for other species (25).

Interestingly, the sequence of strain C7-rat was indistinguishable from that of $B$. elizabethae. As this result was unex-

TABLE 3. Levels of similarity between glt $A$ sequences of $B$. henselae and $B$. quintana strains

\begin{tabular}{|c|c|c|c|c|c|c|c|}
\hline \multirow[b]{2}{*}{ Strain } & \multicolumn{7}{|c|}{$\%$ Similarity } \\
\hline & 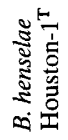 & 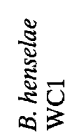 & 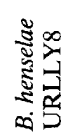 & 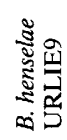 & 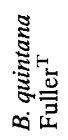 & 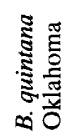 & 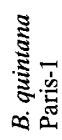 \\
\hline B. henselae WC1 & 99.8 & & & & & & \\
\hline B. henselae URLLY8 & 99.9 & 99.9 & & & & & \\
\hline B. henselae URLIE9 & 99.8 & 100 & 99.9 & & & & \\
\hline B. quintana Fuller ${ }^{T}$ & 91.0 & 91.0 & 91.0 & 91.0 & & & \\
\hline B. quintana Oklahoma & 91.1 & 91.1 & 91.1 & 91.1 & 99.9 & & \\
\hline B. quintana Paris-1 & 91.1 & 91.0 & 91.0 & 91.0 & 99.8 & 99.9 & \\
\hline B. quintana Marseille-1 & 91.1 & 91.0 & 91.0 & 91.0 & 99.8 & 99.9 & 100 \\
\hline
\end{tabular}

pected, the identities of the isolates were confirmed in two ways. First, partial $16 \mathrm{~S}$ rRNA gene and glt $A$ sequences determined for multiple samples of both C7-rat and B. elizabethae matched the sequences of original preparations used in this study. Second, a restriction endonuclease analysis of PCRamplified $16 \mathrm{~S} / 23 \mathrm{~S}$ intragenic spacer regions (25) of C7-rat and $B$. elizabethae revealed profiles which were very similar but distinguishable (data not shown).

The glt $A$ sequences of strains C1-phy, C4-phy, and R-phy2 exhibited levels of similarity of more than $99.6 \%$ with each other, but levels of similarity of less than $93.5 \%$ with the sequences of all other strains. The remaining uncharacterized isolates exhibited less than $93.5 \%$ similarity with all other $\mathrm{Bar}$ tonella strains.

A phylogenetic tree (Fig. 1) was constructed from the distance matrix. Although the genus Bartonella is monophyletic and $B$. bacilliformis is the most outlying species in this group, the architecture of this tree differs markedly from the architecture of the tree derived from 16S rDNA data.

Although the alpha subdivision representative Rickettsia prowazekii is close to the Bartonella cluster, Rhizobium tropici is in a position markedly more distant than the position inferred from the 16S rRNA gene sequence comparison. However, the sequence data available for Rhizobium tropici were derived from glt $A$ in a plasmid rather than genomic DNA (21) and as such cannot be considered representative of the evolution of the species. Although the relationship between the Bartonella species and the species not included in the alpha subdivision inferred from $g l t A$ sequence data is markedly different from the relationship derived from $16 \mathrm{~S}$ rDNA analysis, there is no statistical support for its architecture. From the tree derived from the parsimony analysis (Fig. 2), the instability of the proposed extra-Bartonella architecture was confirmed, as a different 
branching order, again without the support of the bootstrap analysis, was produced.

However, unlike the results obtained in the $16 \mathrm{~S}$ rDNA analysis, the intra-Bartonella phylogeny derived from glt $A$ data has some statistical significance. The following four well-supported ( $>90 \%$ of bootstrap samples) clusters were identified in the genus: (i) consistent with the 16S rDNA analysis data, a unique, relatively deep-rooted divergence of $B$. bacilliformis, (ii) a cluster containing $B$. grahamii, $B$. elizabethae, and the two Peruvian rat isolates, (iii) a cluster containing the four isolates obtained from Phyllotis mice, and (iv) the cluster containing $B$. henselae and $B$. quintana (supported by distance matrix data but not by the results of a parsimony analysis of bootstrap samples).

Previous studies have shown that different small mammal species that inhabit the same geographical location are susceptible to infection by the same Bartonella species and that different Bartonella species can infect the same species of small mammal (4). However, there is clear evolutionary divergence not only between the strains isolated from Phyllotis mice and the strains isolated from rats in the Huaillacayán Valley, but also between $B$. grahamii and the other United Kingdom small mammal isolates. Why such divergence between these strains occurred is not clear; however, species of small mammals are probably infected by the same Bartonella species by sharing the same flea fauna, which serves as a vector for the bacteria. As several different species of fleas are naturally found as ectoparasites on small mammals (26), infection by different Bartonella species may originate from different flea species, with each Bartonella species exhibiting species specificity for its vector. Such a proposal clearly needs further investigation. Interestingly, isolates obtained from Phyllotis mice in the geographically remote Rimac Valley shared a specific relationship with isolates obtained from the Huaillacayán Valley; isolates of $B$. grahamii and $B$. taylorii have also been obtained from geographically distinct populations of small mammals in the United Kingdom (14).

All members of the genus Bartonella except $B$. bacilliformis exhibit very similar and significant levels of DNA-DNA homology $(5,7,8)$. An analysis of the evolutionary distances derived from a glt $A$ comparison of these species revealed that these distances are also very similar, with a narrow standard deviation $(0.024)$ about a mean of $0.132 K_{\text {nuc }}$ (range, between 51 and $121 \%$ of the mean). In contrast, evolutionary distances derived from a 16S rRNA gene comparison exhibit a far broader standard deviation of 0.004 about a mean of 0.009 (range, between 33 and $176 \%$ of the mean). This comparison suggests that glt $A$-derived data are more compatible with DNA-DNA homology results than data derived from $16 \mathrm{~S}$ rRNA genes are. However, such an observation is not unexpected, as the evolutionary distances between Bartonella species inferred from 16S rRNA comparisons are so small that minute differences appear to be significant. The evolutionary distances between Bartonella species inferred from gltA comparisons are 14.7 times greater (mean) than the evolutionary distances derived from 16S rRNA genes; thus, slight differences in distances between species are not very influential. Previous studies of other genera of bacteria have also revealed apparent disagreements between data derived from DNA-DNA homology studies and data derived from comparisons of very similar $16 \mathrm{~S}$ rRNA gene sequences $(14,18)$, and such reports have culminated in a recent taxonomic proposal suggesting that meaningful taxonomic conclusions cannot be confidently inferred from comparisons of sequences that exhibit more than $97 \%$ sequence homology (29).

Furthermore, although the gltA sequences of different Bar- tonella species have diverged at a considerably faster rate than 16S rRNA gene sequences have diverged, the apparent rates of divergence of the two gene sequences in closely related strains appear to be similar. Indeed, B. elizabethae and strain C7-rat, despite having identical glt $A$ sequences, are separated by a branch length similar to the branch length observed between different species on the tree inferred from the $16 \mathrm{~S}$ rRNA gene comparison. Which of these two models is the most accurate is unclear; however, this example demonstrates again that data derived from sequences which are virtually identical cannot be relied on for accurate phylogenetic reconstructions. It could also be proposed that C7-rat and $B$. elizabethae share glt $A$ sequences as a result of a recent transfer of genetic material between the organisms. Such a proposal again highlights the potential problems associated with phylogenetic inferences derived from a comparison of only a single gene.

The correspondence of $B$. elizabethae and C7-rat glt $A$ sequences is unexpected as the former organism has been encountered on only one occasion in the United States (8). Although additional comparisons of these organisms are clearly necessary, it is likely that C7-rat is a strain of B. elizabethae. This finding provides the first evidence that $B$. elizabethae infection, like $B$. henselae infection, is a zoonosis. The high levels of homology among C4-phy, R-phy2, and C1-phy (separated by less than $0.04 K_{\text {nuc }}$, whereas the closest two Bartonella species are $0.66 K_{\text {nuc }}$ apart) suggest that these strains may also belong to a single species. The relationship of the remaining uncharacterized isolates, C5-rat, R-phy1, and N40, to other Bartonella species remains unclear; in particular, N40 exhibits no specific relationship with any of the recognized species or other uncharacterized strains.

Intra-Bartonella phylogeny remains confusing, and it is likely that this problem will become more complicated as additional isolates are obtained and additional characterization studies are performed. Bartonella-like organisms have been observed in the erythrocytes of a wide range of animals (31), and thus it is likely a large number of strains and species have yet to be identified and characterized. However, whereas 16S rDNAbased phylogenies must be considered of little use at this level, data derived from comparisons of glt $A$ sequences have resulted in the identification of some distinct evolutionary divergence within the genus. Extended analysis of the gltA gene from a greater number of isolates should help improve our understanding of the evolutionary relationships in the genus Bartonella. It would also be interesting to assess the utility of this approach for determining the phylogenetic relationships between Bartonella species and other members of the alpha subdivision of the Proteobacteria.

\section{REFERENCES}

1. Ball, M. L. 1994. M.Sc. thesis. Liverpool School of Tropical Medecine, University of Liverpool, Liverpool, United Kingdom.

2. Birtles, R. J. 1995. Differentiation of Bartonella species by restriction endonuclease analysis of PCR-amplified 16S rRNA genes. FEMS Microbiol. Lett. 129:261-266.

3. Birtles, R. J., T. G. Harrison, N. K. Fry, N. A. Saunders, and A. G. Taylor. 1991. Taxonomic considerations of Bartonella bacilliformis based on phylogenetic and phenotypic characteristics. FEMS Microbiol. Lett. 83:187-192.

4. Birtles, R. J., T. G. Harrison, and D. H. Molyneux. 1994. Grahamella in small woodland mammals in the UK: isolation, prevalence and host specificity. Ann. Trop. Med. Parasitol. 88:317-327.

5. Birtles, R. J., T. G. Harrison, N. A. Saunders, and D. H. Molyneux. 1995. Proposals to unify the genera Grahamella and Bartonella, with descriptions of Bartonella talpae comb. nov., Bartonella peromysci comb. nov., and three new species, Bartonella grahamii sp. nov., Bartonella taylorii sp. nov., and Bartonella doshiae sp. nov. Int. J. Syst. Bacteriol. 45:1-8.

6. Breitschwerdt, E. B., D. L. Kordick, D. E. Malarkey, B. Keene, T. L. Hadfield, and K. Wilson. 1995. Endocarditis in a dog due to infection with a novel Bartonella subspecies. J. Clin. Microbiol. 33:154-160.

7. Brenner, D. J., S. P. O'Connor, W. W. Winkler, and A. G. Steigerwalt. 1993. 
Proposals to unify the genera Bartonella and Rochalimaea, with descriptions of Bartonella quintana comb. nov., Bartonella vinsonii comb. nov., Bartonella henselae comb. nov., and Bartonella elizabethae comb. nov., and to remove the family Bartonellaceae from the order Rickettsiales. Int. J. Syst. Bacteriol. 43:777-786.

8. Daly, J. S., M. G. Worthington, D. J. Brenner, C. W. Moss, D. G. Hollis, R. S. Weyant, A. G. Steigerwalt, R. E. Weaver, M. I. Daneshvar, and S. P. O'Connor. 1993. Rochalimaea elizabethae sp. nov. isolated from a patient with endocarditis. J. Clin. Microbiol. 31:872-881.

9. Donald, L. J., and H. W. Duckworth. 1987. Expression and base sequence of the citrate synthase gene of Acinetobacter anitratum. Biochem. Cell Biol.65: 930-938.

10. Drancourt, M., R. J. Birtles, G. Chaumentin, F. Vandenesch, J. Etienne, and D. Raoult. 1996. New serotype of Bartonella henselae in endocarditis and cat-scratch disease. Lancet 347:441-443.

11. Drancourt, M., J. L. Mainardi, P. Brouqui, F. Vandenesch, A. Carta, F. Lehert, J. Etienne, F. Goldstein, J. Acar, and D. Raoult. 1995. Bartonella (Rochalimaea) quintana endocarditis in homeless men. N. Engl. J. Med. 332:419-423.

12. Embley, T. M. 1991. The linear PCR: a simple and robust method for sequencing amplified rRNA genes. Lett. Appl. Microbiol. 13:13-24.

13. Felsenstein, J. 1989. PHYLIP: phylogeny inference package. Cladistics 5:164-166.

14. Fox, G. E., J. D. Wisotzkey, and P. Jurtshuk, Jr. 1992. How close is close: 16S rRNA sequence identity may not be sufficient to guarantee species identity. Int. J. Syst. Bacteriol. 42:166-170.

15. Higgins, D., A. Bleasby, and R. Fuchs. 1992. Clustal V: improved software for multiple sequence analysis. Comput. Appl. Biosci. 8:188-192.

16. Joblet, C. V. Roux, M. Drancourt, J. Gouvernet, and D. Raoult. 1995 Identification of Bartonella (Rochalimaea) species among fastidious gramnegative bacteria on the basis of the partial sequence of the citrate synthase gene. J. Clin. Microbiol. 33:1879-1883.

17. Ludwig, W., J. Neumaier, N. Klugbauer, E. Brockmann, C. Roller, S. Jilg, K. Reetz, I. Schachtner, A. Ludvigsen, M. Bachleitner, U. Fischer, and K. H. Schleifer. 1993, Phylogenetic relationships of bacteria based on comparative sequence analysis of elongation factor Tu and ATP-synthase beta-subunit genes. Antonie van Leeuwenhoek 64:285-305.

18. Martinez-Murcia, A. J., S. Benlloch, and M. D. Collins. 1992. Phylogenetic interrelationships of members of the genera Aeromonas and Plesiomonas as determined by ribosomal 16S rRNA sequencing: lack of congruence with results of DNA-DNA hybridization. Int. J. Syst. Bacteriol. 42:412-421.

19. Ner, S. S., V. Bhayana, A. W. Bell, I. G. Giles, H. W. Duckworth, and D. P.
Bloxham. 1983. Complete sequence of the gltA gene encoding citrate synthase in Escherichia coli. Biochemistry 22:5243-5249.

20. Norman, A. F., R. Regnery, P. Jameson, C. Greene, and D. C. Krause. 1995 Differentiation of Bartonella-like isolates at the species level by PCR-restriction fragment length polymorphism in the citrate synthase gene. J. Clin. Microbiol. 33:1797-1803.

21. Pardo, M. A., J. Lagunez, J. Miranda, and E. Martinez. 1994. Nodulating ability of Rhizobium tropici is conditioned by a plasmid-encoded citrate synthase. Mol. Microbiol. 11:315-321.

22. Raoult, D., M. Drancourt, A. Carta, and J. A. Gastaut. 1994. Letter. Lancet 343:977.

23. Regnery, R. L., B. E. Anderson, J. E. Clarridge III, M. C. RodriguezBarradas, D. C. Jones, and J. H. Carr. 1992. Characterization of a novel Rochalimaea species, $R$. henselae sp. nov., isolated from blood of a febrile, human immunodeficiency virus-positive patient. J. Clin. Microbiol. 30:265274.

24. Regnery, R. L., C. L. Spruill, and B. D. Plikaytis. 1991. Genotypic identification of rickettsiae and estimation of intraspecies sequence divergence for portions of two rickettsial genes. J. Bacteriol. 173:1576-1589.

25. Roux, V., and D. Raoult. 1995. Inter- and intraspecies identification of Bartonella (Rochalimaea) species. J. Clin. Microbiol. 33:1573-1579.

26. Smit, F. G. A. M. 1957. Handbooks for the identification of British insects, vol. 1. Royal Entomological Society of London, London.

27. Sneath, P. H. A. 1993. Evidence from Aeromonas for genetic crossing-over in ribosomal sequences. Int. J. Syst. Bacteriol. 43:626-629.

28. Springer, E., M. S. Sachs, C. R. Woese, and D. R. Boone. 1995. Partial gene sequences for the A subunit of methyl-coenzyme $M$ reductase $(m c r l)$ as a phylogenetic tool for the family Methanosarcinaceae. Int. J. Syst. Bacteriol. 45:554-559.

29. Stackebrandt, E., and B. M. Goebel. 1994. Taxonomic note: a place for DNA-DNA reassociation and $16 \mathrm{~S}$ rRNA sequence analysis in the present species definition in bacteriology. Int. J. Syst. Bacteriol. 44:846-849.

30. Stein, A., and D. Raoult. 1992. A simple method for amplification of DNA from paraffin-embedded tissues. Nucleic Acids Res. 20:5237-5238.

31. Weinmann, D., and J. P. Kreier. 1977. Bartonella and Grahamella, p. 197 233. In J. P. Kreier (ed.), Parasitic protozoa, vol. 4. Academic Press, London.

32. Wiegand, G., and S. J. Remington. 1986. Citrate synthase: structure, control, and mechanism. Annu. Rev. Biophys. Biophys. Chem. 15:97-117.

33. Wood, D. O., L. R. Williamson, H. H. Winkler, and D. C. Krause. 1987. Nucleotide sequence of the Rickettsia prowazekii citrate synthase gene. J. Bacteriol. 169:3564-3572. 